\title{
Preterm Infant Outcomes Following COVID-19 Lockdowns in Melbourne, Australia
}

\author{
Brendan Mulcahy ${ }^{1}$, Daniel L. Rolnik ${ }^{2,3}{ }^{(0)}$, Alexia Matheson ${ }^{3}$, Yizhen Liu ${ }^{2,3}$, Kirsten R. Palmer ${ }^{2,3} \mathbb{C}^{\text {, }}$ \\ Ben W. Mol ${ }^{3,4}$ and Atul Malhotra ${ }^{1,5, *(1)}$ \\ 1 Monash Newborn, Monash Children's Hospital, Melbourne, VIC 3168, Australia; \\ mulcahy.brendan@gmail.com \\ 2 Department of Obstetrics and Gynaecology, Monash University, Melbourne, VIC 3168, Australia; \\ daniel.rolnik@monash.edu (D.L.R.); ayzliu@gmail.com (Y.L.); kirsten.palmer@monash.edu (K.R.P.) \\ 3 Department of Obstetrics and Gynaecology, Monash Health, Melbourne, VIC 3168, Australia; \\ alexiawmatheson@gmail.com (A.M.); ben.mol@monash.edu (B.W.M.) \\ 4 Aberdeen Centre for Women's Health Research, School of Medicine, University of Aberdeen, \\ Aberdeen AB24 3FX, UK \\ 5 Department of Paediatrics, Monash University, Melbourne, VIC 3168, Australia \\ * Correspondence: atul.malhotra@monash.edu
}

check for

updates

Citation: Mulcahy, B.; Rolnik, D.L.; Matheson, A.; Liu, Y.; Palmer, K.R.; Mol, B.W.; Malhotra, A. Preterm Infant Outcomes Following COVID-19 Lockdowns in Melbourne, Australia. Children 2021, 8, 1169. https://doi.org/10.3390/ children 8121169

Academic Editor: Jörn-Hendrik Weitkamp

Received: 13 November 2021 Accepted: 8 December 2021 Published: 10 December 2021

Publisher's Note: MDPI stays neutral with regard to jurisdictional claims in published maps and institutional affiliations.

Copyright: (c) 2021 by the authors. Licensee MDPI, Basel, Switzerland. This article is an open access article distributed under the terms and conditions of the Creative Commons Attribution (CC BY) license (https:// creativecommons.org/licenses/by/ $4.0 /)$.

\begin{abstract}
Background Community lockdowns during the coronavirus disease 2019 (COVID-19) pandemic may influence preterm birth rates, but mechanisms are unclear. Methods We compared neonatal outcomes of preterm infants born to mothers exposed to community lockdowns in 2020 (exposed group) to those born in 2019 (control group). Main outcome studied was composite of significant neonatal morbidity or death. Results Median gestational age was $35+4$ weeks (295 infants, exposed group) vs. $35+0$ weeks (347 infants, control group) $(p=0.108)$. The main outcome occurred in 36/295 (12.2\%) infants in exposed group vs. 46/347 (13.3\%) in control group ( $p=0.69)$. Continuous positive airway pressure (CPAP) use, jaundice requiring phototherapy, hypoglycaemia requiring treatment, early neonatal white cell and neutrophil counts were significantly reduced in the exposed group. Conclusions COVID-19 community lockdowns did not alter composite neonatal outcomes in preterm infants, but reduced rates of some common outcomes as well as early neonatal inflammatory markers.
\end{abstract}

Keywords: coronavirus; inflammatory markers; morbidity; mortality; newborn

\section{Introduction}

The coronavirus disease 2019 (COVID-19) pandemic has had a far-reaching impact on maternal and infant wellbeing [1,2]. While COVID-19 infection in pregnancy has been associated with an increased risk of preterm birth [3,4], several studies have suggested a reduction in preterm births in non-infected mothers during community lockdown [5-9]. Very few studies have assessed what impact this has had on neonatal outcomes $[10,11]$, and previous studies are from countries with relatively high COVID-19 community transmission rates.

Melbourne, Australia, experienced one of the world's strictest community transmission mitigation measures during two lockdowns (March-May, July-November) in 2020. These measures coupled with relatively low rates of community transmission then make us uniquely placed to examine the effects of lockdown measures on neonatal outcomes. Furthermore, some studies have suggested reduced exposure to infective pathogens as a reason for reduced preterm birth during lockdown [5-8]. We aimed to explore effects of community lockdowns on neonatal outcomes of preterm infants, with a secondary focus on the underlying rates of systemic inflammation in infants and mothers around the time of birth. 


\section{Methods}

We conducted a retrospective cohort study at Monash Health, Melbourne's largest public health service ( $>10,000$ births per year). Data were gathered from the network's birth registry and from electronic medical records. Ethical approval was obtained from Monash Health Human Research Ethics Committee (approval number QA/69113/MonH2020-235157). Preterm infants ( $>20$ weeks and $<37$ weeks' gestation) born to mothers who conceived between 1 November 2019 and 29 February 2020, who were between 3 and 19 weeks pregnant at the beginning of lockdown (exposed group), compared to preterm infants conceived at the same time period the year before (control group) were included [3]. Conception was calculated based on gestational age from first day of the last menstrual period and confirmed with first trimester ultrasound.

We compared neonatal outcomes of preterm infants born during lockdown in 2020 (exposed group) with preterm infants born in 2019 (control group). The main outcome studied was a composite of death or significant morbidity in preterm infants, including chronic lung disease (CLD), severe (grade III-IV) intraventricular haemorrhage (IVH), necrotising enterocolitis (NEC), retinopathy of prematurity (ROP) requiring treatment, or culture positive sepsis. The secondary outcomes were rates of individual preterm complications and differences in inflammatory markers between cohorts.

For comparison of inflammatory markers we chose infants born $<34$ weeks, along with their mothers, due to local hospital protocol to screen these infants for infection. Included were first neonatal C-reactive protein (CRP) and full blood count (FBC) within seven days of birth, and last maternal CRP and FBC prior to delivery. Neonatal total white cell count (WCC), neutrophil count and immature-to-total neutrophil (IT) ratio were recorded. Categorical variables were compared using chi-squared or Fisher's Exact test. Continuous variables were evaluated for normality of distribution. Non-normally distributed continuous variables were compared using the Wilcoxon rank-sum test and normally distributed variables using independent-samples t-tests. Effect estimates are reported as odds ratios (OR) of exposed compared to controls with $95 \%$ confidence intervals (CI). $p$ values below 0.05 were considered statistically significant. Analyses were performed using Stata statistical package (Release 16, College Station, TX, USA).

\section{Results}

There were 295/3187 (9.3\%) live neonates born $<37$ weeks in the exposed group, versus $347 / 3229(10.7 \%$ ) live neonates in the control group (OR 0.85; 95\% CI 0.72-1.00; $p=0.047$ ). Median gestational age was $35+4$ weeks in the exposed group (IQR $33+1$ to $35+3$ weeks) compared with $35+0$ weeks ( $32+3$ to $36+2$ weeks) in the control group $(p=0.108)$.

The primary outcome occurred in $36(12.2 \%)$ infants $<37$ weeks in exposed group, compared with 46 (13.3\%) infants $<37$ weeks in control group (OR 0.91; 95\% CI 0.57-1.45; $p=0.69$ ). When the mother was considered the unit of analysis, the primary outcome due to prematurity occurred in $36 / 3187(1.1 \%)$ versus $46 / 3229(1.4 \%)$ of live neonates born before 37 weeks in the exposed group and non-exposed group (RR $0.79,95 \%$ CI 0.51 to 1.20$)$.

Secondary outcomes showed a significant reduction in continuous positive airway pressure (CPAP) requirement, jaundice requiring phototherapy, hypoglycaemia requiring treatment, admission to the neonatal intensive care unit (NICU) $>48 \mathrm{~h}$ and median NICU length of stay in the exposed group (Table 1). There were no significant differences in other individual outcomes between the groups, including neonatal death.

Significant reductions in neonatal white cell counts $(9.2$, IQR $6.2-12.0$ vs. 10.7, IQR 7.9-13.8; $p=0.01$ ) and neutrophil counts (3.1, IQR 1.8-4.9 vs. 3.8, IQR 2.3-5.7; $p=0.04$ ) were seen in the exposed group compared to controls. There were no significant differences in maternal inflammatory markers (Table 2). 
Table 1. Comparison of neonatal outcomes (live born neonates $<37$ weeks gestation).

\begin{tabular}{|c|c|c|c|c|}
\hline Outcomes & $\begin{array}{l}\text { Exposed Group } \\
(n=295)\end{array}$ & $\begin{array}{l}\text { Unexposed Group } \\
\qquad(\mathrm{n}=347)\end{array}$ & $\begin{array}{c}\text { Odds Ratio or Mean } \\
\text { Difference } \\
(95 \% \mathrm{CI})\end{array}$ & $p$ Value \\
\hline Gestational Age, median (IQR) & $35^{+4}\left(33^{+1}-35^{+3}\right)$ & $35^{+0}\left(32^{+3}-36^{+2}\right)$ & - & 0.108 \\
\hline Birth weight (g), mean (SD) & $2219(763)$ & $2094(736)$ & $125(8-242)$ & 0.004 \\
\hline Primary composite outcome ${ }^{\$}$ & $36(12.2)$ & $46(13.3)$ & $0.91(0.57-1.45)$ & 0.690 \\
\hline Chronic lung disease & $15(5.1)$ & $25(7.2)$ & $0.69(0.36-1.33)$ & 0.268 \\
\hline IVH grade III or IV & $7(2.7)$ & $3(0.9)$ & $2.79(0.71-10.88)$ & 0.124 \\
\hline Necrotising enterocolitis & $2(0.7)$ & $7(2.0)$ & $0.33(0.07-1.61)$ & 0.150 \\
\hline Retinopathy of prematurity & $4(1.4)$ & $2(0.6)$ & $2.37(0.43-13.0)$ & 0.306 \\
\hline Culture-positive sepsis & $8(2.7)$ & $15(4.3)$ & $0.62(0.26-1.48)$ & 0.274 \\
\hline RDS requiring intubation & $25(8.5)$ & $28(8.1)$ & $1.05(0.60-1.85)$ & 0.850 \\
\hline CPAP requirement & $66(22.4)$ & $114(32.9)$ & $0.59(0.41-0.84)$ & 0.003 \\
\hline Jaundice requiring phototherapy & $110(37.3)$ & $165(47.6)$ & $0.66(0.48-0.90)$ & 0.009 \\
\hline Hypoglycaemia requiring IVT & $59(20.0)$ & $108(31.1)$ & $0.55(0.38-0.80)$ & 0.001 \\
\hline Osteopenia requiring treatment & $17(5.8)$ & $33(9.5)$ & $0.58(0.32-1.07)$ & 0.077 \\
\hline PDA requiring treatment & $9(3.1)$ & $14(4.0)$ & $0.75(0.32-1.76)$ & 0.504 \\
\hline Neonatal seizures & $2(0.7)$ & $2(0.6)$ & $1.18(0.16-8.41)$ & 0.871 \\
\hline Hyponatraemia & $18(6.1)$ & $26(7.5)$ & $0.80(0.43-1.49)$ & 0.487 \\
\hline Hypernatraemia & $9(3.1)$ & $16(4.6)$ & $0.65(0.28-1.50)$ & 0.309 \\
\hline Admission to NICU $>48 \mathrm{~h}$ & $82(27.8)$ & $127(36.6)$ & $0.67(0.48-0.93)$ & 0.018 \\
\hline Median NICU stay, days (IQR) & $5(0-21)$ & $9(0-27)$ & - & 0.019 \\
\hline Neonatal death & $11(3.7)$ & $8(2.3)$ & $1.64(0.65-4.14)$ & 0.289 \\
\hline
\end{tabular}

Data presented as a total number (percentage) unless specified. CI: confidence interval; CPAP: Continuous positive airway pressure; GA: gestational age; IQR: Interquartile range; IVH: intraventricular haemorrhage; IVT: intravenous therapy; LBW: low birth weight; NICU: Neonatal Intensive Care Unit; PDA: patent ductus arteriosus; RDS: respiratory distress syndrome; VLBW: very low birth weight. $\$$ Primary composite outcome $=$ death or significant neonatal morbidity (chronic lung disease, severe IVH grade III or IV, necrotising enterocolitis, retinopathy of prematurity requiring treatment, culture positive sepsis, RDS requiring intubation).

Table 2. Comparison of inflammatory markers among preterm neonates and mothers who gave birth to a preterm neonate ( $<34$ weeks gestation).

\begin{tabular}{cccc}
\hline Inflammatory Markers & $\begin{array}{c}\text { Exposed Group } \\
(\mathbf{n}=\mathbf{9 0})\end{array}$ & $\begin{array}{c}\text { Unexposed Group } \\
(\mathbf{n}=\mathbf{1 2 9})\end{array}$ & $p$-Value \\
Maternal results & & & \\
C-reactive protein, in $\mathrm{mg} / \mathrm{L}$ & $12.0(6.0-41.0)$ & $17.0(7.0-27.0)$ & $13.8(12.0-17.2)$ \\
White cell count, in $10^{9} / \mathrm{L}$ & $13.5(10.8-16.0)$ & $10.5(8.7-13.9)$ & 0.924 \\
Neutrophil count, in $10^{9} / \mathrm{L}$ & $10.4(8.2-12.9)$ & $1 / 106(0.9)$ & 0.193 \\
Blood culture + ve sepsis, $n(\%)$ & $1 / 74(1.4)$ & & 0.302 \\
Neonatal results & & $0.2(0-0.7)$ & 0.991 \\
C-reactive protein, in $\mathrm{mg} / \mathrm{L}$ & $0.2(0-0.8)$ & $10.7(7.9-13.8)$ & 0.011 \\
White cell count, in $10^{9} / \mathrm{L}$ & $9.2(6.2-12.0)$ & $3.8(2.3-5.7)$ & 0.041 \\
Neutrophil count, in $10^{9} / \mathrm{L}$ & $3.1(1.8-4.9)$ & $0.2(0.1-0.7)$ & 0.478 \\
Immature cells, in $10^{9} / \mathrm{L}$ & $0.3(0.1-0.7)$ & $0.06(0.02-0.14)$ & $0.019 *$ \\
Immature-to-total neutrophil ratio & $0.10(0.05-0.17)$ & $6(4.7)$ & 0.943 \\
Blood culture + ve sepsis, $n(\%)$ & $4(4.4)$ & & \\
\hline
\end{tabular}

Data presented as median (IQR 25th-75th percentile). IQR: interquartile range. * Denominator is number of pregnant women who delivered at least one live infant $<34$ weeks. ${ }^{* *}$ Although significantly different between groups, both ranges are within normal limits.

\section{Discussion}

While we found a $15 \%$ reduction in preterm births $<37$ weeks gestation in the lockdown cohort, there was no significant difference in the composite primary neonatal outcome among preterm infants. Seasonal variability in preterm births was not assessed, given both cohorts encompassed the same time period in adjacent calendar years.

Among preterm infants, there was a 33\% reduction in the odds of needing NICU admission, a $41 \%$ reduction in CPAP requirement, a $34 \%$ reduction in neonatal jaundice 
requiring phototherapy and a $45 \%$ reduction in hypoglycaemia requiring intravenous therapy in the exposed group. The reduction in these common neonatal complications could reflect the shift seen towards a more mature gestational age and significantly higher birth weight (mean $125 \mathrm{~g}$ heavier) of infants in the exposed group as compared to unexposed group. Ours is the first study that we are aware of to report on as comprehensive a range of neonatal outcomes following lockdown measures, particularly in light of the reduction in premature births observed previously [6]. There are limited studies that reported on neonatal outcomes following lockdown [11,12]. They did not show any statistically significant reductions in individual neonatal outcomes. The authors of a systematic review of perinatal outcomes during the pandemic era were only able to report comparable data for three outcome measures (five-minute Apgar scores $<7$, birth weight $<2500 \mathrm{~g}$ and NICU admission) to include in their meta-analysis, due to a lack of studies assessing any other specific neonatal outcomes [13]. Our results are almost the exact opposite of the findings from studies of infants born to mothers acutely affected by COVID-19, in whom an increase in NICU admission, respiratory distress and jaundice has been observed [10], reinforcing that our observations are likely due to the impact of community lockdown alone in a maternal population without high community COVID-19 infection rates.

This study is the first published evidence we are aware of to link lockdown to a possible reduction in inflammatory markers in the neonatal period. We found a $14 \%$ and $18 \%$ reduction in neonatal total white cell counts and neutrophil counts, respectively, for preterm infants born of mothers exposed to lockdown. It is plausible that the reduction of preterm birth rates in lockdown is at least in part due to reduced infant exposure to inflammation processes, as had been hypothesised previously [5-8]. A reduction in other common viral infections has indeed been noticed during COVID-19 community lockdowns, which may be contributory to decreased inflammation observed in the perinatal period [14-16]. Future studies could consider assessment of other inflammatory markers such as the NLRP3 inflammasome that have been identified as having a role in the pathogenesis of clinical disease in COVID-19 infection [17]. Furthermore, the role of altered placental inflammation may be important to explore in the future.

Strengths of this study include it being the first to assess such a broad range of neonatal outcomes in relation to lockdown measures. Limitations include the retrospective design and the relatively small numbers of preterm infants affected by significant morbidity, limiting the statistical power. Future studies should also explore the role of maternal stress on preterm birth and outcomes in populations exposed to community lockdowns.

\section{Conclusions}

Within Melbourne's stringent COVID-19 lockdowns and low burden of community COVID-19 spread, we found no difference in significant morbidity or death between groups, but saw a reduction in preterm births $<37$ weeks, CPAP requirement, jaundice and hypoglycaemia among preterm infants born to mothers exposed to community lockdowns. A reduction in neonatal white cell counts and neutrophil counts was also seen in the exposed cohort. Further investigation is needed to confirm these findings and how they may be used to direct future lifestyle interventions that may reduce inflammation in pregnancy and help reduce prematurity rates.

Author Contributions: Conceptualization, D.L.R., B.W.M., A.M. (Atul Malhotra); methodology, D.L.R., B.W.M., A.M. (Atul Malhotra); software, B.M., D.L.R.; validation, D.L.R., A.M. (Atul Malhotra); formal analysis, B.M., D.L.R.; investigation, B.M., D.L.R., A.M. (Alexia Matheson), Liu, Y., Palmer, K.R., B.W.M. and A.M. (Atul Malhotra); resources, D.L.R., B.W.M., A.M. (Atul Malhotra); data curation, B.M., D.L.R., A.M. (Alexia Matheson), Liu, Y., Palmer, K.R. ,B.W.M. and A.M. (Atul Malhotra); writing—original draft preparation, B.M.; writing—review and editing, B.M., D.L.R., A.M. (Alexia Matheson), Liu, Y., Palmer, K.R. ,B.W.M. and A.M. (Atul Malhotra); visualization, A.M. (Atul Malhotra); supervision, A.M. (Atul Malhotra); project administration, D.L.R., B.W.M., A.M. (Atul Malhotra); funding acquisition, N.A. All authors have read and agreed to the published version of the manuscript. 
Funding: No funding was received for this study.

Institutional Review Board Statement: Ethical approval was obtained from Monash Health Human Research Ethics Committee (approval number QA/69113/MonH-2020-235157).

Informed Consent Statement: De-identified group data presented. Individual consent not required for this observational study.

Data Availability Statement: Data available on reasonable request.

Conflicts of Interest: The authors declare no conflict of interest.

\section{References}

1. Roberton, T.; Carter, E.D.; Chou, V.B.; Stegmuller, A.R.; Jackson, B.D.; Tam, Y.; Sawadogo-Lewis, T.; Walker, N. Early estimates of the indirect effects of the COVID-19 pandemic on maternal and child mortality in low-income and middle-income countries: A modelling study. Lancet Glob. Health 2020, 8, e901-e908. [CrossRef]

2. Burki, T. The indirect impact of COVID-19 on women. Lancet Infect. Dis. 2020, 2, 904-905. [CrossRef]

3. Knight, M.; Bunch, K.; Vousden, N.; Morris, E.; Simpson, N.; Gale, C.; O’Brien, P.; Quigley, M.; Brocklehurst, P.; Kurinczuk, J.J. Characteristics and outcomes of pregnant women admitted to hospital with confirmed SARS-CoV-2 infection in UK: National population based cohort study. BMJ 2020, 369, m2107. [CrossRef] [PubMed]

4. Pierce-Williams, R.A.M.; Burd, J.; Felder, L.; Khoury, R.; Bernstein, P.S.; Avila, K.; Penfield, C.A.; Roman, A.S.; DeBolt, C.A.; Stone, J.L.; et al. Clinical course of severe and critical coronavirus disease 2019 in hospitalized pregnancies: A United States cohort study. Am. J. Obs. Gynecol. MFM 2020, 2, 100134. [CrossRef] [PubMed]

5. Hedermann, G.; Hedley, P.L.; Bækvad-Hansen, M.; Hjalgrim, H.; Rostgaard, K.; Poorisrisak, P.; Breindahl, M.; Melbye, M.; Hougaard, D.M.; Christiansen, M.; et al. Danish premature birth rates during the COVID-19 lockdown. Arch. Dis. Child Fetal Neonatal Ed. 2021, 106, 93-95. [CrossRef] [PubMed]

6. Rolnik, D.L.; Matheson, A.; Liu, Y.; Chu, S.; Mcgannon, C.; Mulcahy, B.; Malhotra, A.; Palmer, K.R.; Hodges, R.J.; Mol, B.W. The impact of COVID-19 pandemic restrictions on pregnancy duration and outcomes in Melbourne, Australia. Ultrasound Obstet. Gynecol. 2021, 58, 677-687. [CrossRef] [PubMed]

7. Philip, R.K.; Purtill, H.; Reidy, E.; Daly, M.; Imcha, M.; McGrath, D.; O'Connell, N.H.; Dunne, C.P. Unprecedented reduction in births of very low birthweight (VLBW) and extremely low birthweight (ELBW) infants during the COVID-19 lockdown in Ireland: A 'natural experiment' allowing analysis of data from the prior two decades. BMJ Glob. Health 2020, 5, e003075. [CrossRef]

8. Been, J.V.; Burgos Ochoa, L.; Bertens, L.C.M.; Schoenmakers, S.; Steegers, E.A.P. Reiss IKM. Impact of COVID-19 mitigation measures on the incidence of preterm birth: A national quasi-experimental study. Lancet Public Health 2020, 5, e604-e611. [CrossRef]

9. Matheson, A.; McGannon, C.J.; Malhotra, A.; Palmer, K.R.; Stewart, A.E.; Wallace, E.M.; Mol, B.W.; Hodges, R.J.; Rolnik, D.L. Prematurity Rates During the Coronavirus Disease 2019 (COVID-19) Pandemic Lockdown in Melbourne, Australia. Obs. Gynecol. 2021, 137, 405-407. [CrossRef]

10. Norman, M.; Navér, L.; Söderling, J.; Ahlberg, M.; Hervius, A.H.; Aronsson, B.; Byström, E.; Jonsson, J.; Sengpiel, V.; Ludvigsson, J.F.; et al. Association of Maternal SARS-CoV-2 Infection in Pregnancy With Neonatal Outcomes. JAMA 2021, 325, 2076-2086. [CrossRef] [PubMed]

11. Meyer, R.; Bart, Y.; Tsur, A.; Yinon, Y.; Friedrich, L.; Maixner, N.; Levin, G. A marked decrease in preterm deliveries during the coronavirus disease 2019 pandemic. Am. J. Obs. Gynecol. 2021, 224, 234-237. [CrossRef] [PubMed]

12. Mor, M.; Kugler, N.; Jauniaux, E.; Betser, M.; Wiener, Y.; Cuckle, H.; Maymon, R. Impact of the COVID-19 Pandemic on Excess Perinatal Mortality and Morbidity in Israel. Am. J. Perinatol. 2021, 38, 398-403. [CrossRef] [PubMed]

13. Chmielewska, B.; Barratt, I.; Townsend, R.; Kalafat, E.; Meulen, J.V.D.; Gurol-Urganci, I.; O’Brien, P.; Morris, E.; Draycott, T.; Thangaratinam, S.; et al. Effects of the COVID-19 pandemic on maternal and perinatal outcomes: A systematic review and meta-analysis. Lancet Glob. Health 2021, 9, e759-e772. [CrossRef]

14. Yeoh, D.K.; Foley, D.A.; Minney-Smith, C.A.; Martin, A.C.; Mace, A.O.; Sikazwe, C.T.; Le, H.; Levy, A.; Blyth, C.C.; Moore, H.C. The impact of COVID-19 public health measures on detections of influenza and respiratory syncytial virus in children during the 2020 Australian winter. Clin. Infect. Dis. 2020, 72, 2199-2202. [CrossRef] [PubMed]

15. Kuitunen, I.; Artama, M.; Mäkelä, L.; Backman, K.; Heiskanen-Kosma, T.; Renko, M. Effect of Social Distancing Due to the COVID-19 Pandemic on the Incidence of Viral Respiratory Tract Infections in Children in Finland During Early 2020. Pediatr. Infect. Dis. J. 2020, 39, e423-e427. [CrossRef] [PubMed]

16. Marriott, D.; Beresford, R.; Mirdad, F.; Stark, D.; Glanville, A.; Chapman, S.; Harkness, J.; Dore, G.J.; Andresen, D.; Matthews, G.V. Concomitant marked decline in prevalence of SARS-CoV-2 and other respiratory viruses among symptomatic patients following public health interventions in Australia: Data from St Vincent's Hospital and associated screening clinics, Sydney, NSW. Clin. Infect. Dis. 2020, 72, e649-e651. [CrossRef] [PubMed]

17. Sergi, C.M.; Chiu, B. Targeting NLRP3 inflammasome in an animal model for Coronavirus Disease 2019 (COVID-19) caused by the Severe Acute Respiratory Syndrome Coronavirus 2 (SARS-CoV-2). J. Med. Virol. 2021, 93, 669-670. [CrossRef] [PubMed] 